\title{
Patient-specific 3-dimensionally printed models for neurosurgical planning and education
}

\author{
Sandip S. Panesar, MD, MSc, ${ }^{1}$ Michael Magnetta, MD, MS, ${ }^{2}$ Debraj Mukherjee, MD, MPH, ${ }^{3}$ \\ Kumar Abhinav, MD, ${ }^{4}$ Barton F. Branstetter, MD, ${ }^{5}$ Paul A. Gardner, MD, ${ }^{6}$ Michael Iv, MD, ${ }^{7}$ and \\ Juan C. Fernandez-Miranda, MD ${ }^{4}$
}

\begin{abstract}
'Department of Neurosurgery, Houston Methodist Hospital, Houston, Texas; ${ }^{2}$ Department of Radiology, Northwestern University, Chicago, Illinois; ${ }^{3}$ Department of Neurosurgery, Johns Hopkins University, Baltimore, Maryland; Departments of ${ }^{4}$ Neurosurgery and ${ }^{7}$ Radiology, Stanford University, Stanford, California; and Departments of ${ }^{5}$ Radiology and ${ }^{6}$ Neurological Surgery, University of Pittsburgh, Pittsburgh, Pennsylvania
\end{abstract}

OBJECTIVE Advances in 3-dimensional (3D) printing technology permit the rapid creation of detailed anatomical models. Integration of this technology into neurosurgical practice is still in its nascence, however. One potential application is to create models depicting neurosurgical pathology. The goal of this study was to assess the clinical value of patientspecific 3D printed models for neurosurgical planning and education.

METHODS The authors created life-sized, patient-specific models for 4 preoperative cases. Three of the cases involved adults ( 2 patients with petroclival meningioma and 1 with trigeminal neuralgia) and the remaining case involved a pediatric patient with craniopharyngioma. Models were derived from routine clinical imaging sequences and manufactured using commercially available software and hardware.

RESULTS Life-sized, 3D printed models depicting bony, vascular, and neural pathology relevant to each case were successfully manufactured. A variety of commercially available software and hardware were used to create and print each model from radiological sequences. The models for the adult cases were printed in separate pieces, which had to be painted by hand, and could be disassembled for detailed study, while the model for the pediatric case was printed as a single piece in separate-colored resins and could not be disassembled for study. Two of the models were used for patient education, and all were used for presurgical planning by the surgeon.

CONCLUSIONS Patient-specific 3D printed models are useful to neurosurgical practice. They may be used as a visualization aid for surgeons and patients, or for education of trainees.

https://thejns.org/doi/abs/10.3171/2019.9.FOCUS19511

KEYWORDS 3D printing; stereolithography; medical modeling; educational aids; operative planning

$\mathrm{M}$ ASTERING the complex 3-dimensional (3D) neuroanatomy of the cranium, parenchyma, and their vascular supply represents a significant learning curve for the trainee neurosurgeon. Surgical neuroanatomy is often learned from texts, 2D images, or postmortem specimens, none of which offer an exact representation of natural variations or pathological distortions. Currently, preoperative planning utilizes data acquired from MRI and CT sequences, from which surgeons may plan their approaches, with or without image guidance. ${ }^{3,9}$ While novel 3D reconstruction and visualization methods (such as virtual reality $[\mathrm{VR}]^{7,16,19}$ and augmented reality $[\mathrm{AR}]^{11,16}$ ) are being increasingly utilized, conventional radiological imaging usually only provides a $2 \mathrm{D}$, cross-sectional view of anatomy for the surgeon. ${ }^{6}$ Advancements in additive manufacturing (3D printing) allow the creation of complex, 3D objects using segmentation software, computeraided design (CAD) software, and a 3D printer. ${ }^{12,22}$ For the neurosurgeon, potential clinical applications of 3D printing include the design of custom prostheses, ${ }^{2,14}$ the production of educational models, ${ }^{1,4}$ and the creation of training simulators. ${ }^{21,23,24}$ Additionally, 3D printing allows the creation of patient-specific, life-sized, and accurate models that may be used prior to surgery in order to visualize anatomy and pathology and plan surgical approaches. ${ }^{4}$ Furthermore, these models may be used in the operating

ABBREVIATIONS AR = augmented reality; $C N$ = cranial nerve; FIESTA = fast imaging employing steady-state acquisition; TE = echo time; TR = repetition time; VR = virtual reality.

SUBMITTED June 28, 2019. ACCEPTED September 5, 2019

INCLUDE WHEN CITING DOI: 10.3171/2019.9.FOCUS19511. 
room, where they can provide surgeons with a real-time, patient-specific anatomical reference during surgery.

The growing prevalence of affordable 3D printing software and hardware has promoted technological uptake and exploration of its clinical uses. Various institutions across the United States have established "clinical 3D printing" facilities as subsidiaries of clinical departments. These permit clinicians from various specialties to utilize fabrication services for obtaining rapidly fabricated clinical models, which are then used for surgical planning, teaching, or patient education. Nevertheless, as the technology is nascent, the clinical benefits and overall remit of clinical 3D printing have yet to be fully defined. Here, we present a series of 3 adult and 1 pediatric neurosurgical cases for which we rapidly fabricated preoperative 3D models, using similar 3D printing services at 2 institutions. Standard clinical radiological sequences were used to create each model, and commercially available software and hardware were used to fabricate the models. Our series is intended to serve as a demonstration of the capabilities of 3D printing, in the hope that we can promote its uses and further promote its integration into clinical practice.

\section{Methods}

This study was approved by institutional review boards at the University of Pittsburgh and Stanford University. All patients in the included cases consented to the use of their data for this case series.

\section{Cases}

\section{Case 1: Petroclival Meningioma}

A 67-year-old man presented as an outpatient with a 2-year history of intermittent dizziness and a 2-month history of left-sided numbness, with occasional pain along the trigeminal distribution. Pertinent past medical history included nonmetastatic Gleason stage 6 prostate carcinoma. Examination elicited numbness along the V1-3 distributions of the left trigeminal nerve. Initial neuroimaging revealed a $35 \times 20 \times 40-\mathrm{mm}$ left-sided, dural-based meningioma within the posterior fossa. As part of routine presurgical workup, the patient underwent both unenhanced and contrast-enhanced MRI scans of the brain and CT angiography of the head with stereotactic localization (Fig. 1).

\section{Case 2: Petroclival Meningioma}

A 58-year-old woman presented to the outpatient clinic with a 9-month history of bladder incontinence, unsteadiness, fatigue, and mild right-sided facial drooping. Her past medical history was unremarkable and physical examination was noncontributory. Imaging studies revealed a $37 \times$ $34 \times 50-\mathrm{mm}$ petroclival meningioma. As part of her routine presurgical workup she underwent both unenhanced and contrast-enhanced MRI of the brain and CT angiography of the head for stereotactic localization (Fig. 2).

\section{Case 3: Trigeminal Neuralgia Secondary to Vascular Impingement}

A 67-year-old woman presented with a 4-year history of right-sided, sudden-onset, stabbing, paroxysmal facial pain in the V3 distribution. There was chronic background V3 soreness and she underwent a tooth-extraction procedure without relief of the pain. Her pain was initially medically managed with carbamazepine, but she could not tolerate increased doses used to counter an increasing frequency of attacks. An open retromastoid microvascular decompression was scheduled for treatment of trigeminal neuralgia secondary to vascular impingement. Prior to surgery she underwent standard neuroimaging sequences and a noncontrast fast imaging employing steady-state acquisition (FIESTA) MRI sequence (Fig. 3). This case was added subsequently as a feasibility study of 3D printing very small structures, including the brainstem vasculature and cranial nerves.

\section{Case 4: Pediatric Craniopharyngioma}

A 2-year-old boy presented with seizures, intractable vomiting, and diabetes insipidus. Imaging at an outside hospital revealed a lesion consistent with craniopharyngioma invading the sellar and suprasellar regions. He initially underwent a stereotactic biopsy and cyst fenestration shortly after diagnosis, before presenting for definitive treatment. After extensive surgical planning, using CT and MRI and a 3D printed model, he underwent lesion resection and insertion of a right frontal external ventricular drain via an extended endoscopic endonasal (transtuberculum transplanum) approach, which was performed in conjunction with otolaryngology specialists. Skull base reconstruction was performed primarily using a nasoseptal flap. He woke up neurologically intact after resection of the lesion (Fig. 4).

\section{Image Acquisition \\ Cases 1 and 2}

We used DICOM images taken from preoperative CT angiography with stereotactic localization. Scanning was performed on a GE LightSpeed VCT 64-slice CT scanner (GE Healthcare) at $120 \mathrm{kVp}, 185-225 \mathrm{~mA}$, and pitches ranging from 0.52 to 1.0 . The images were reconstructed in $1.25-\mathrm{mm}$ axial sections using standard reconstruction and $30 \%$ adaptive statistical iterative reconstruction (ASIR, GE Healthcare). The DICOM images were anonymized and exported from the picture archiving and communication system (PACS) and sent to a secure DICOM receiver in the $3 \mathrm{D}$ printing laboratory at the University of Pittsburgh.

\section{Case 3}

For case 3, the noncontrast FIESTA sequence was obtained using a GE Discovery MRI system (GE Healthcare) with a 3.0-T magnet. Images were acquired using 1.0-mmthick slabs and $0.5-\mathrm{mm}$ spaces. The repetition time (TR) was $5.685 \mathrm{msec}$ and echo time (TE) was $2.063 \mathrm{msec}$, with a flip angle of $65.0^{\circ}$.

\section{Case 4}

This case utilized both CT and MRI sequences, which were used together to create a model with bony, vascular, and neural structures. CT angiography was performed on the Siemens SOMATOM Definition Flash CT scan- 

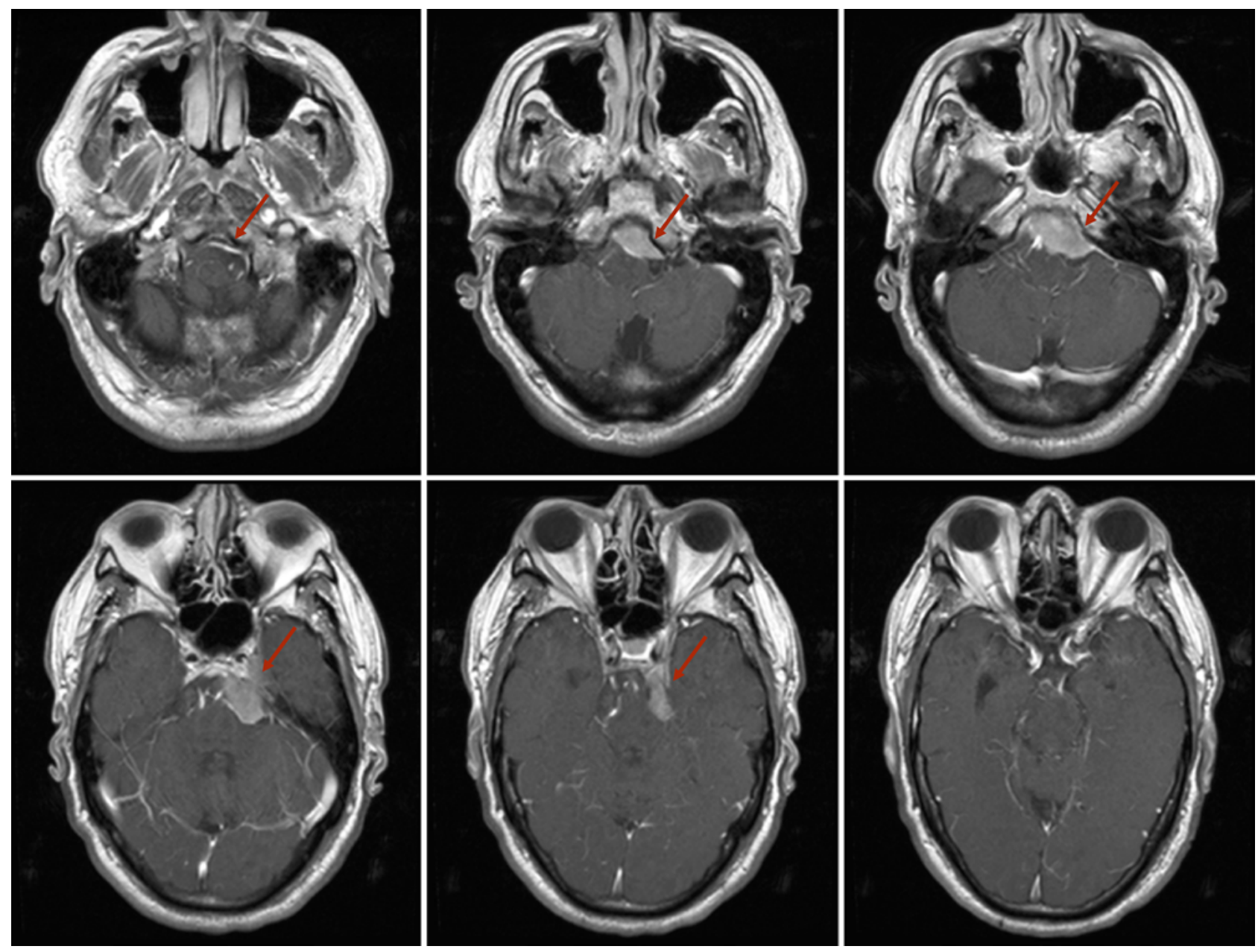

FIG. 1. Case 1. Sequential axial contrast-enhanced T1-weighted MR images (inferosuperior view) depicting pathology and vascular structures. In each image, the red arrow indicates the lesion, a dural-based petroclival meningioma (with a dural tail), which is compressing vascular and parenchymal structures.

ner (Siemens Healthineers) with the following scan parameters: 1-mm slice thickness, 0.5 -mm slice spacing, 80 $\mathrm{kVp}$, and 300-500 mAs. The scanning was triggered by the CT technologist based on contrast enhancement of the aortic arch following the intravenous administration of iohexol with a concentration of $350 \mathrm{mg} / \mathrm{mL}$ (Omnipaque 350 , GE Healthcare) at a rate of 4 to $5 \mathrm{~mL} / \mathrm{sec}$.

MRI was performed on a 3-T scanner (MR Discovery 750, GE Healthcare) equipped with a standard 8-channel head coil. Precontrast axial spoiled gradient recalled echo-brain volume (SPGR-BRAVO) images were obtained using the following parameters: TR $10.4 \mathrm{msec}$, TE $4.3 \mathrm{msec}$, slice thickness $1 \mathrm{~mm}$, slice spacing $1 \mathrm{~mm}$, matrix $224 \mathrm{~mm} \times 224 \mathrm{~mm}$, field of view $220 \mathrm{~mm}$, inversion time $450 \mathrm{msec}$, and flip angle $15^{\circ}$.

\section{Virtual Model Creation}

\section{Cases 1-3}

DICOM images were loaded into Mimics Medical v20.0 (Materialise, Inc.), where segmentation of the tumor, osseous structures of the skull, and adjacent surgical anatomy of interest was performed by threshold and manual segmentation operations. For tumors, the threshold operation kept pixel values with Hounsfield units of contrast-enhanced tumor and excluded all other values. The tumor was then isolated from adjacent structures by manually removing unwanted structures from the segmentation. The same operation was then performed for osseous skull structures and vasculature, as necessary for each model. Separate 3D models of the tumor, osseous, and vascular structures were then computed and exported to 3-Matic Medical v12.0 (Materialise, Inc.), where mesh optimization, smoothing, and mesh repair were performed. For models that showcased anatomy better with removable parts, magnet attachment sites were incorporated into the model. The largest piece of each model was then labeled with the corresponding PACS accession number and a stereolithography (.stl) file of the model was exported. Modeling each case took varying lengths of time, ranging from 4 to 8 hours.

\section{Case 4}

DICOM CT angiography and MR images were loaded into TeraRecon Aquarius Intuition Workstation v4.4.13.136, where bony, vascular, and neural structures 

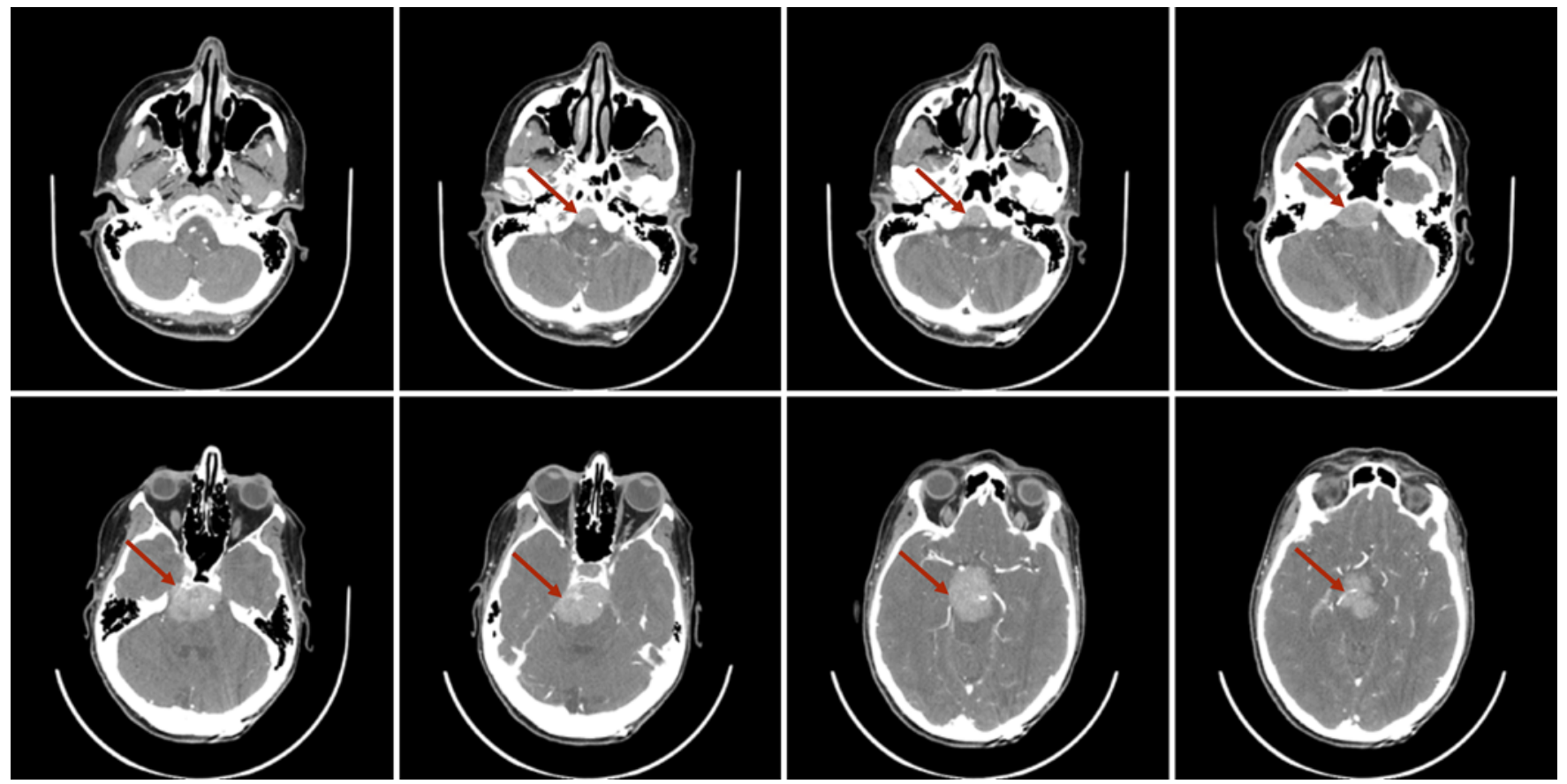

FIG. 2. Case 2. Sequential axial contrast-enhanced CT images (inferosuperior view). Red arrows indicate tumor consistent with petroclival meningioma. Surrounding vascular structures are readily visible.

were segmented into .stl format. Following segmentation, the .stl model was loaded into Materialise 3Matic v21.1 for optimization prior to 3D printing. For this case, visualization of the optic nerve and chiasm was crucial for preoperative planning. We utilized the MR sequence in order to visualize and segment these neural structures. The model for case 5 was printed in its entirety using a printer with the capability of printing different colors together; therefore, a single .stl model was utilized, as opposed to the models for cases $1-4$, for which the bony and nonbony structures were printed separately. Creating the virtual model for this case took approximately 2 hours.

\section{D Printed Model Fabrication}

\section{Cases 1-3}

The .stl files were loaded into PreForm (Formlabs Inc.) and placed in an optimal configuration according to Formlabs guidelines. An axial print resolution of $0.1 \mathrm{~mm}$ was
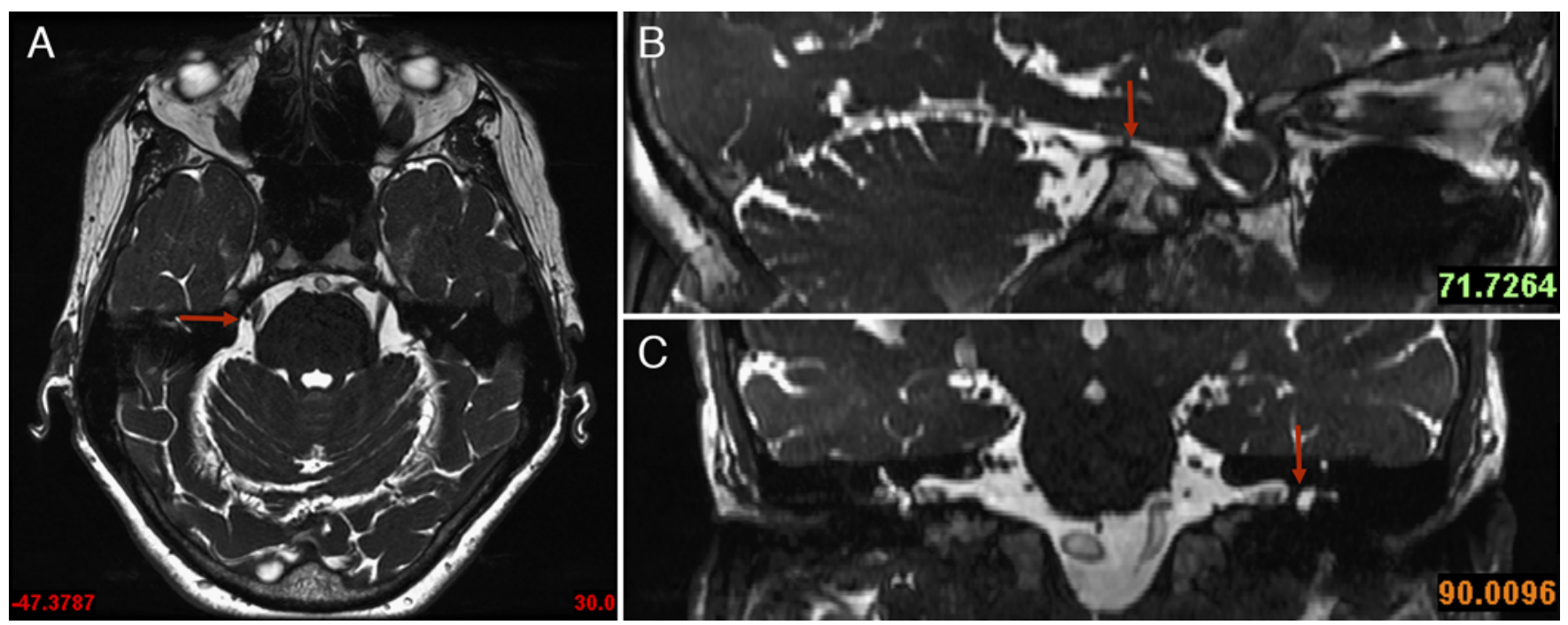

FIG. 3. Case 3. FIESTA MR images demonstrating axial (A), sagittal (B), and coronal (C) views of the cisternal portions of CN V with vascular impingement by the superior cerebellar artery (red arrows). 

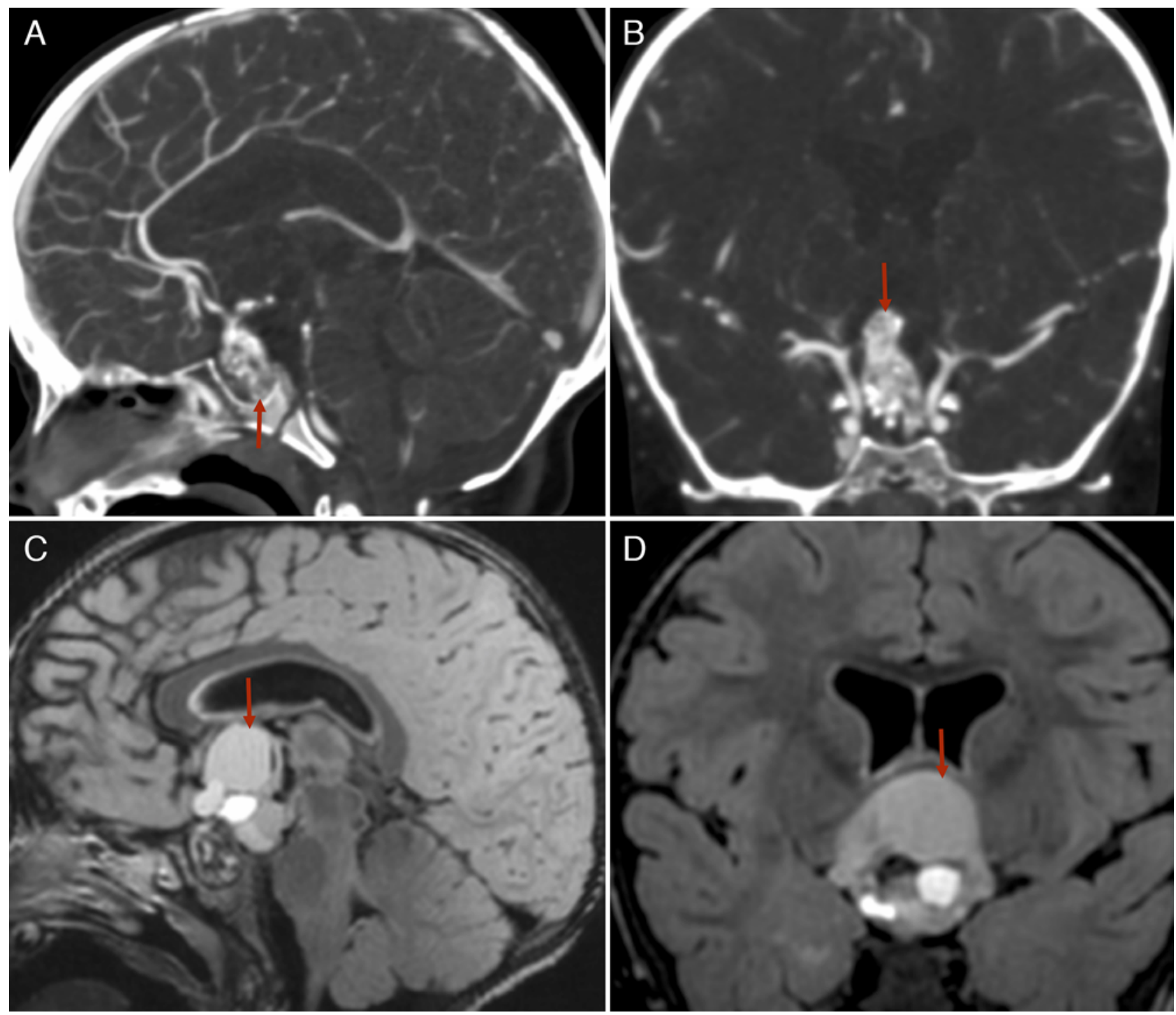

FIG. 4. Case 4. A and B: Sagittal (A) and coronal (B) contrast-enhanced CT images. Red arrows indicate tumor mass consistent with a craniopharyngioma. C and D: Sagittal (C) and coronal (D) MR images of the tumor structure (indicated by red arrows).

selected in white acrylic photosensitive resin (Formlabs Inc.) for osseous structures and clear acrylic photosensitive resin for tumor and vascular structures. Support features were automatically added to support the model during the printing process. The support locations were edited such that no support material contacted delicate anatomy or regions of interest. The print file was then saved and sent to a Form 2 stereolithography (SLA) 3D printer (Formlabs Inc.). The model preparation times ranged from 4 to 6 hours depending on complexity and feature engineering, such as the addition of magnetic attachment structures. When printing was finished, the model was removed and washed for a total of 10 minutes in a Form Wash (Formlabs Inc.) bath of $99 \%$ isopropyl alcohol to remove any uncured resin from the surface. Supporting structures left over from the printing process were then physically removed. Arterial and venous structures were painted red and blue, respectively. Meningeal and tumor structures were left clear. If the model required neodymium magnets for attachment of removable parts, the magnets were affixed with cyanoacrylate.

\section{Case 4}

The .stl files were loaded into Stratasys GrabCAD (Stratasys Ltd.) for optimization prior to printing. The model was printed using a Stratasys J730 model printer. This is a multimaterial printer, permitting up to 5 differently colored materials to be printed simultaneously. The model was printed in Stratasys Vero, which is a rigid photopolymer available in different colors. Colors chosen for the model were white for osseous structures, yellow for neural structures, red for vascular structures, and green for the tumor. It took approximately 5 hours to print the entire model. Following printing, the model was soaked in water to remove excess material and clean it. Supporting structures were then manually removed. 

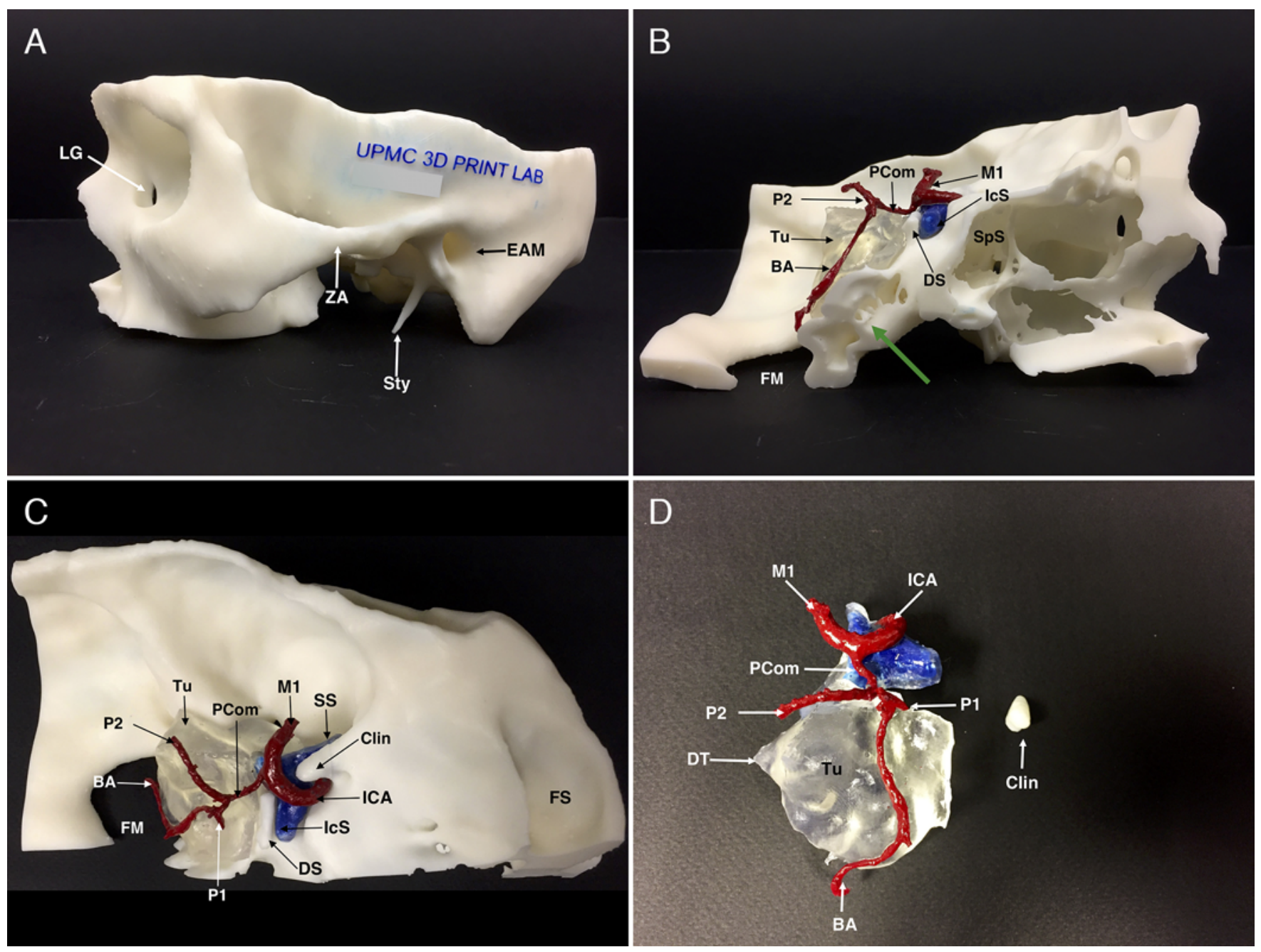

FIG. 5. Case 1. A: Model depicting the cranium and pathology in case 1. This view illustrates the extent of the cranial window from the lateral aspect and several important bony structures including the styloid process (Sty), external auditory meatus (EAM), zygomatic arch (ZA), and lacrimal groove (LG). B: Medial view of the model depicting the pathology in case 1. Visible is the hemisection through the skull in the sagittal plane. The left anterior and middle cranial fossae are depicted from this view, with the foramen magnum (FM) representing the most basal point of the model. Anterior to the sphenoid body lies the sphenoid sinus (SpS), with the bony nasal structures lying anterior to the SpS and clearly depicting bony structures encountered during an endoscopic endonasal approach to the tumor. The tumor (Tu) has been reproduced in clear resin, with associated arterial (red) and venous (blue) structures also visible. Visible arteries include the ascending basilar artery (BA) superficial to the tumor and its intersection with the $\mathrm{P}_{2}$ segment of the posterior cerebral artery (PCA), which connects with the $M_{1}$ segment of the middle cerebral artery $(\mathrm{MCA})$ via the posterior communicating artery (PCom). Venous structures visible from this angle include those traveling within the intercavernous sinus (ICS), bounded posteriorly by the bony dorsum sellae (DS). The green arrow depicts areas of bony defect within the sphenoid body, an artifact from the modeling process not representative of real anatomy. C: Superior view of model depicting pathology in case 1. Visible superiorly are the frontal sinus (FS) and the anterior and middle cranial fossae, with the FM at the inferoposterior extremity of the model. The tumor $(T u)$ is visible clearly from this angle, with overlying $B A$ and $P_{2}$ and $P_{1}$ arterial segments. The PCom communicates with the $\mathrm{M}_{1}$ component of the MCA. The clinoid process (Clin) and arterial structures overly the venous components of the sphenoid sinus (SS) and intercavernous sinus (IcS). D: The clinoid process (Clin), tumor, and vascular structures have been removed from the model. Clearer from this view is the section of the circle of Willis relevant to this tumor, with $\mathrm{P}_{2}, \mathrm{P}_{1}, \mathrm{PCom}, \mathrm{M}_{1}$, and the internal carotid artery (ICA) all clearly visible overlying the tumor. Also depicted clearly in this view is one of the dural tail (DT) components of the tumor.

\section{Results}

Model print time ranged from 3.75 hours for small tumor and vascular structures (case 2) to 18.25 hours for osseous skull structures (case 4). Required consumable resin ranged from $22.28 \mathrm{~mL}$ (US \$3.41) to $271.45 \mathrm{~mL}$ (US \$40.72). Results and descriptions are presented in Figs.
5-8, which demonstrate the finished models with appropriate labeling to highlight anatomical structures and showcase model features.

\section{Discussion}

We have successfully $3 \mathrm{D}$ printed 4 cases of patient-spe- 

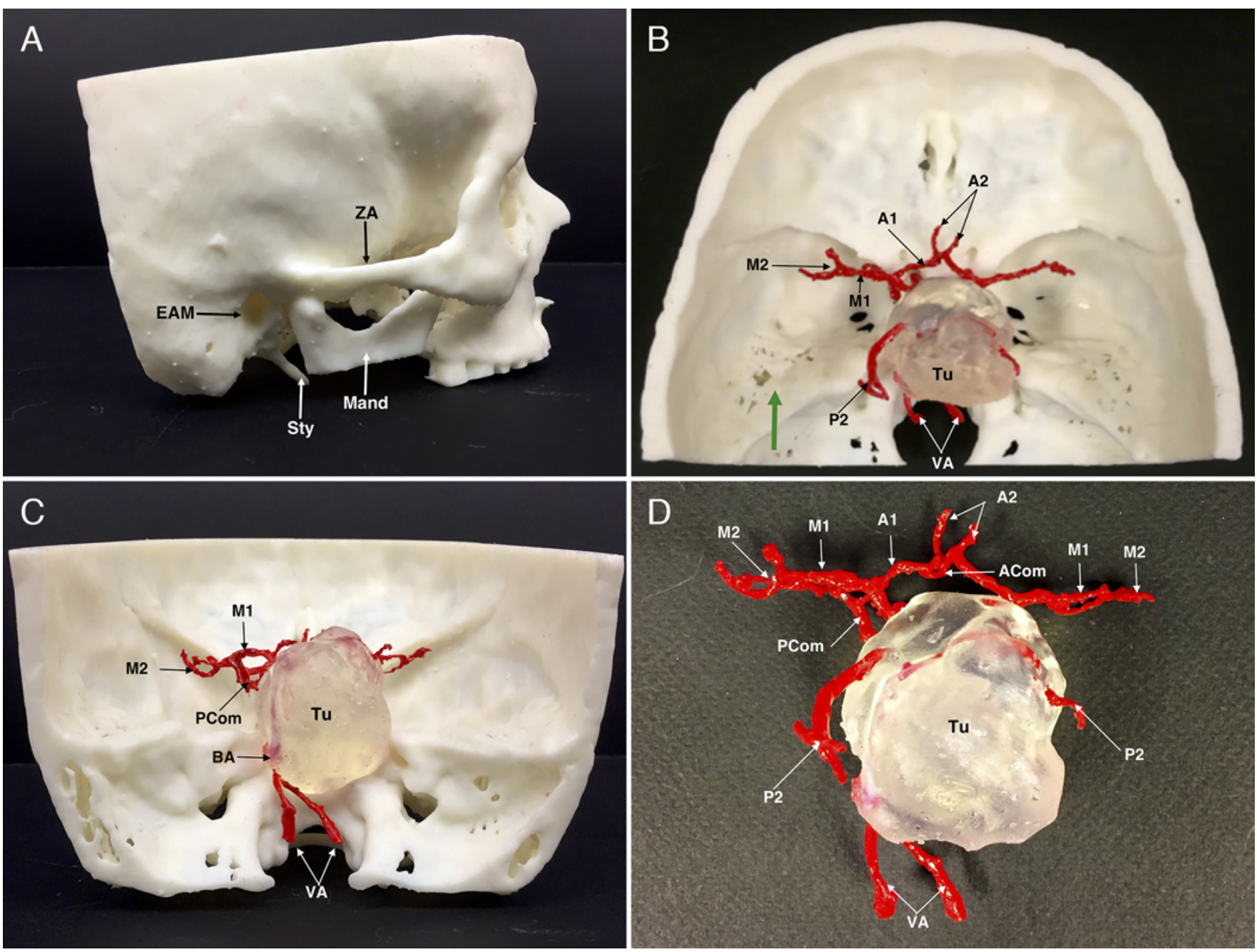

FIG. 6. Case 2. A: A lateral view of the model created for case 2 depicting the superior, inferior, and posterior planar extremities of the model. Visible are the external auditory meatus (EAM), the styloid process (Sty), a portion of the mandible (Mand), and the zygomatic arch (ZA). B: A superoinferior view of the model for case 2, depicting the anterior and middle cranial fossae. The tumor (Tu) lies superior to the clivus (not visible). The arterial vasculature relevant to the tumor is visible, including the $M_{1}$ and $M_{2}$ segments of the MCA on the left and the $A_{1}$ and $A_{2}$ segments of the anterior cerebral artery (ACA). Within the tumor substance originates the $\mathrm{P}_{2}$ segment on the left, itself originating from the BA (not pictured). A portion of each of the vertebral arteries (VAs), ascending via the foramen magnum, is visible. The green arrow indicates an artifactual area of bony defect in the temporal bone (an artifact of the model creation process). C: A posteroanterior coronal view of the model depicting the anterior and middle cranial fossae and peritumoral bony and arterial structures. From this view the vertical size of the lesion is readily apparent, as is its distortion of the circle of Willis vasculature. The BA, lying anterior to the tumor and originating from the VAs, has been significantly displaced to the left and is visible. From this view the horizontal view of the $M_{1}$ and $M_{2} M C A$ branches is also visible, as is the left PCom, arising from the lateral aspect of the tumor. D: The single piece of printed tumor and vascular structures has been removed from the bony cranium to demonstrate the arterial anatomy in detail. Clearly visible are the superior arterial structures, including the $M_{1}$ and $M_{2} M C A$ segments on the left and right, and the $A_{1}$ and $A_{2} A C A$ segments, with the adjoining anterior communicating artery (ACom) centrally. On the left, the PCom is visible, with $\mathrm{P}_{2}$ emerging from the left superolateral aspect of the tumor. On the right, $\mathrm{P}_{2}$ travels from medial to lateral along the superior surface of the tumor before emerging from the right lateral aspect of the tumor. Inferiorly, the VAs join to form the BA.

cific models of neurosurgical anatomy and pathology. All of these models were utilized for preoperative planning by the operating surgeons, and in cases 2 and 4, the models were also utilized for patient education.

\section{Technical Aspects of Medical 3D Printing in Neurosurgery}

All models were created in a time frame that allowed preoperative utilization without delaying surgery. The models were created using DICOM data from conventional preoperative neuroimaging modalities and sequences. Regarding initial virtual model creation, specialized software was required to transform the imaging data into appropriate .stl files for printing. Model creation required specialized hardware, software, and training requirements. ${ }^{18}$ The cost of raw materials required for printing is relatively low in comparison; for our case series, costs for 

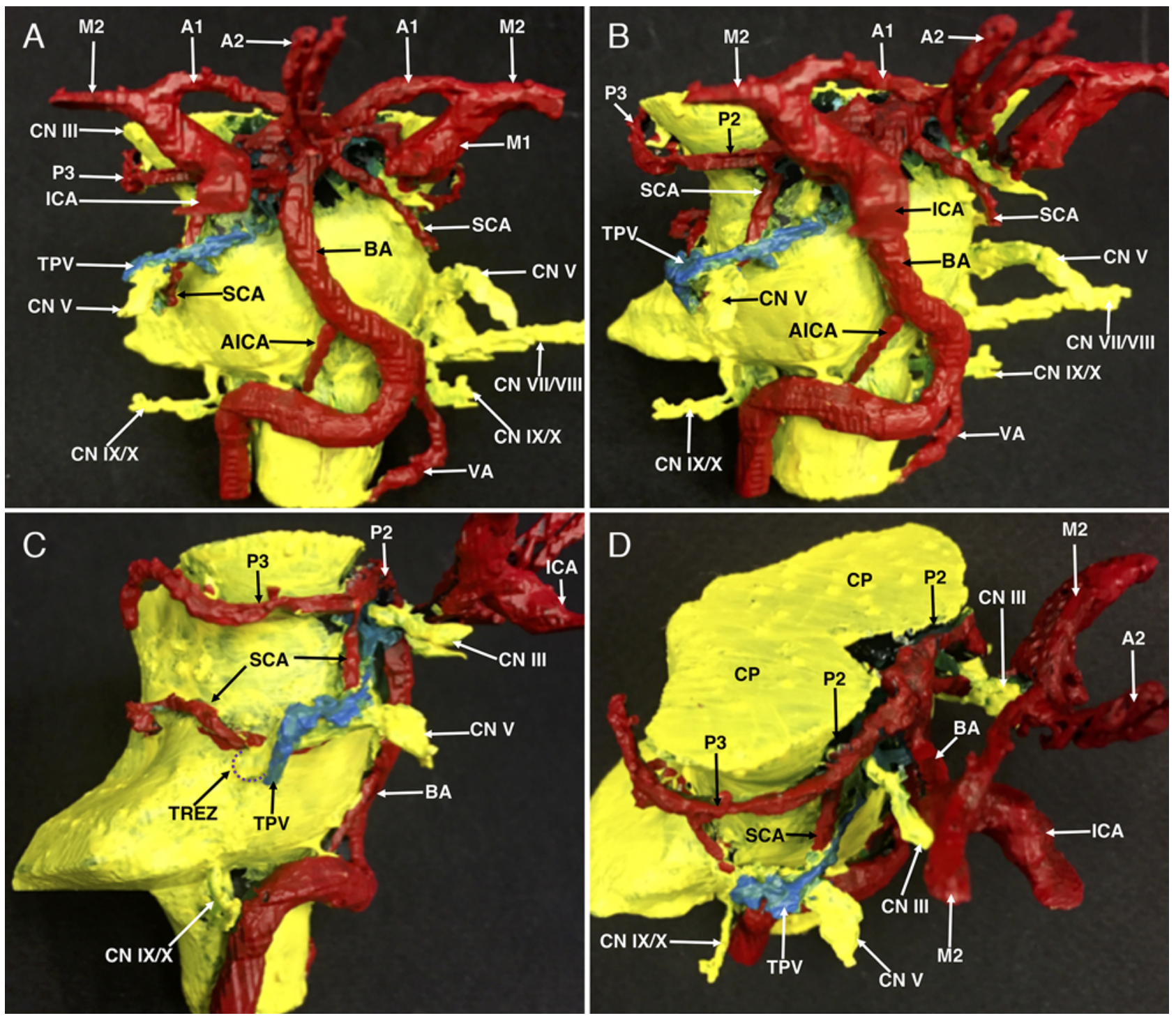

FIG. 7. Case 3. A: Model of brainstem region viewed from an anterior coronal perspective. It demonstrates the pons, medulla, cranial nerves, and overlying vascular structures. Neural tissue, including the cranial nerves, is painted yellow. Cranial nerves depicted include the oculomotor (CN III), trigeminal (CN V), and glossopharyngeal and vagus (CN IX/X) on the right. Cranial nerves visible on the left include the trigeminal (CN V), facial and vestibulocochlear complex (CN VII/VIII), and glossopharyngeal and vagus (CN IX/X). Arterial structures visible include the BA, the VA, and the anterior inferior cerebellar artery (AICA). At the dorsal aspect, a portion of the ICA can be viewed on the right, while on the left the $M_{1}$ segment of the MCA is visible. Bilaterally, the $A_{1}$ segment joins the $M_{1}$ segments, which then continue as $M_{2}$ segments of the MCA. The $A_{1}$ segments continue as the $A_{2}$ segments, which are depicted at the upper center of the picture. Other branches of the BA that are visible from this angle are the left superior cerebellar artery (SCA) and right $\mathrm{P}_{3}$ segment of the PCA. The vein implicated in this case is the transverse pontine vein (TPV), painted blue and visible on the left. B: Model of brainstem region viewed from a right-anterior-oblique perspective. This aspect demonstrates more clearly the impingement upon the trigeminal nerve (CN V) by the right SCA and TPV. Above CN V, SCA, and TPV are the $P_{2}$ (medial) and $P_{3}$ (lateral) segments of the PCA, which wraps around the cerebral peduncle as it travels posteriorly. Anterior to $\mathrm{CN} \mathrm{V}$, on the anterior surface of the pons, is the BA, which gives rise to the AICA inferiorly and itself is formed from the VAs, only one of which is visible. Other contributors to the vasculature of this aspect of the brainstem are the ICA, which continues as the $M_{1}$ (not labeled in this image) and $M_{2}$ segments of the MCA. Medial to $M_{1}$ are the $A_{1}$ segments of the ACA, terminating as the $A_{2}$ branches bilaterally. Neural structures visible on the right include the glossopharyngeal/vagus nerves (CN IX/X). On the left are the trigeminal, facial/vestibulocochlear complex (CN VII/VIII), and glossopharyngeal/vagus nerves (CN IX/X). CN VII and VIII and CN IX and X cannot be differentiated from one another due to their extremely small sizes and the technical difficulties of printing structures of submillimeter sizes. C: Model of brainstem region viewed from a right-sagittal perspective. Visible vascular structures include the $\mathrm{P}_{2}$ and $\mathrm{P}_{3}$ segments of the PCA as it wraps around the inferopeduncular region, traveling posteriorly. Traveling inferiorly, the SCA, another branch of the BA, originates perpendicular to the PCA. After traveling inferiorly, the SCA changes course, turning approximately $90^{\circ}$ to travel posteriorly, parallel to the $\mathrm{P}_{2}$, as it continues posteriorly. It is here that it contacts the trigeminal root entry zone (TREZ), which has been highlighted by placing a broken purple line around its location. Another vascular structure contacting the TREZ is the TPV which has been painted blue. Other visible neural structures are the right oculomotor nerve (CN III) and right glossopharyngeal/vagus nerves (CN IX/X). FIG. 7. (continued) $\rightarrow$ 
FIG. 7. D: A superior-oblique view of the brainstem from a right-anterior perspective. This aspect demonstrates the coronal level where the cerebral peduncles (CPs) were cut to create the model. Arterial structures visible anteriorly are the bilateral $\mathrm{P}_{2}$ segments of the PCAs, which originate from the $B A$. The right $P_{2}$ wraps around the peduncle to continue as the $P_{3}$. Immediately ventral to the PCAs, the oculomotor nerves (CN III) arise bilaterally. Other arterial structures visible anteriorly are a portion of the ICA and the $M_{2}$ segment of the MCA on the right, and the $M_{2}$ and $A_{2}$ segments of the MCA and ACA, respectively. The SCA arises from the BA inferiorly to the PCA, traveling downward along with the TPV, and the TPV and SCA contact the trigeminal nerve (CN V) at different areas, with the SCA causing impingement near the trigeminal root, and the TPV contacting closer to Meckel's cave. Other neural structures visible are the glossopharyngeal/vagus nerves on the right.

raw materials ranged from approximately $\$ 3.00$ to $\$ 40.00$ per case. Technology acquisition expenses and training requirements represent the greatest barrier to widespread institutional implementation of 3D printing for preoperative planning. Another potential technical issue is the extent of visualization required from the model.

The model in case 1 is a hemisection of the maxillofacial cranium, whereas in the models produced for cases 2 and 4 other sections of the cranium have been omitted. By printing relevant structures only, the time required for model creation and subsequent printing may be reduced. As is evident in our models, certain areas of the structures depict artifactual bony defects. These may be filled during the modeling process, but doing so will extend model creation time and printing time, and will require increased volumes of resin. Moreover, filling these defects may not be necessary for the model to fulfill its intended purpose.

A further issue comes when considering the diminutive size of certain skull base structures, such as blood vessels and cranial nerves. Even with the use of a high-resolution FIESTA sequence for its creation, the model in case 3 demonstrated "steps" caused by the neural structures being smaller than the minimum voxel size used in the imaging process. Accordingly, the use of alternative methods like high-resolution tractography may be superior to 3D printing for visualization of cranial nerves. Nevertheless, tractography is unable to depict the vascular structures of the skull base.

Regarding creation of the virtual templates from which the models are printed, surgical experience and clinical and anatomical knowledge are prerequisites to ensure that the model accurately depicts relevant neuroanatomical structures in such a way as to be beneficial to the surgeon.

\section{Educational Value in Neurosurgical Training}

Endonasal skull base surgery represents a technical challenge for trainees. ${ }^{20}$ Patient-specific 3D printed models may be used to enhance understanding of particular approaches and relevant anatomical landmarks encountered during endoscopic surgery. They may also be used for spatial reference during surgery. Furthermore, as evidenced by both cases of petroclival meningiomas, tumor distortion of the small arterial structures around the clivus may be readily depicted. These models are therefore beneficial in depicting patient-specific vascular aberrations caused by pathology (see below for neurovascular usage). Replication of the cranial nerve structures requires transformation and overlap of MRI and CT sequences within the same space, adding complexity and time to the model creation and printing process. To address this challenge, we have conducted preliminary work into modeling and printing cranial nerve structures (cases 3 and 4). For case
3 , we utilized a harder (albeit more expensive) resin to cast this model because of the delicacy of the cranial nerves. We were able to model cranial nerve $(\mathrm{CN}) \mathrm{V}$ and its impingement by the superior cerebellar artery, but the process of modeling, casting, and painting the structures was extremely time consuming. Although the pertinent neurovascular structures are visualized, including the areas of vascular contact with $\mathrm{CN}$ V, detail is still lacking. For case 4 , we successfully managed to demonstrate displacement of the optic nerve and chiasm by the craniopharyngioma. Moreover, due to the hardware used, the structures could be readily printed in different colors. Nevertheless, as $\mathrm{CN}$ II is one of the larger cranial nerves, its segmentation was straightforward. For very small structures, the use of a device that is able to print directly in colored resins without postfabrication modification may save time and result in models that are more accurate, overall, than those that have been manually painted.

\section{Future Directions and Alternative Uses}

Development of a neurosurgical simulator is another potential use for 3D modeling in medical education. Particular to simulation are the accurate depiction of anatomical and pathological structures, a lifelike haptic experience, and the ability for inexpensive production or reuse. Initial neurosurgical implementation of 3D simulators has mainly involved cerebrovascular surgery, $8,10,13,27$ with one study demonstrating a benefit of reduced operative times for coiling of arteriovenous malformations after practice on simulators. ${ }^{25}$ Other proposed applications are extraventricular drain placement, ${ }^{21}$ pedicle screw placement, ${ }^{15}$ and craniotomy simulation. ${ }^{23,24}$ Neurosurgery offers multidimensional potential for applying stereolithographic modeling to enhance trainee experience, as evidenced by our present application of this technology. In theory these models may be utilized as "phantoms" for surgeons to actually simulate surgical approaches prior to the real case. Nevertheless, our models only possessed limited anatomical structures, which may not necessarily bear similar tactile and structural properties to real tissues. To achieve a useful neurosurgical simulator, multiple synthetic materials must be utilized. These should provide haptic properties similar to spongy and petrous bone, arteries, veins, and parenchymal tissues in order to provide a realistic experience for the trainee.

Aside from depicting cranial and intracranial anatomy, 3D printing of spinal structures for education or planning or to create prostheses is also an area of active exploration. ${ }^{26}$ Already, 3D printed vertebral prostheses with viable structural augmentation properties have been implanted into humans successfully. ${ }^{17,28}$ Other possible avenues for $3 \mathrm{D}$ printing in spinal surgery include the creation of pa- 


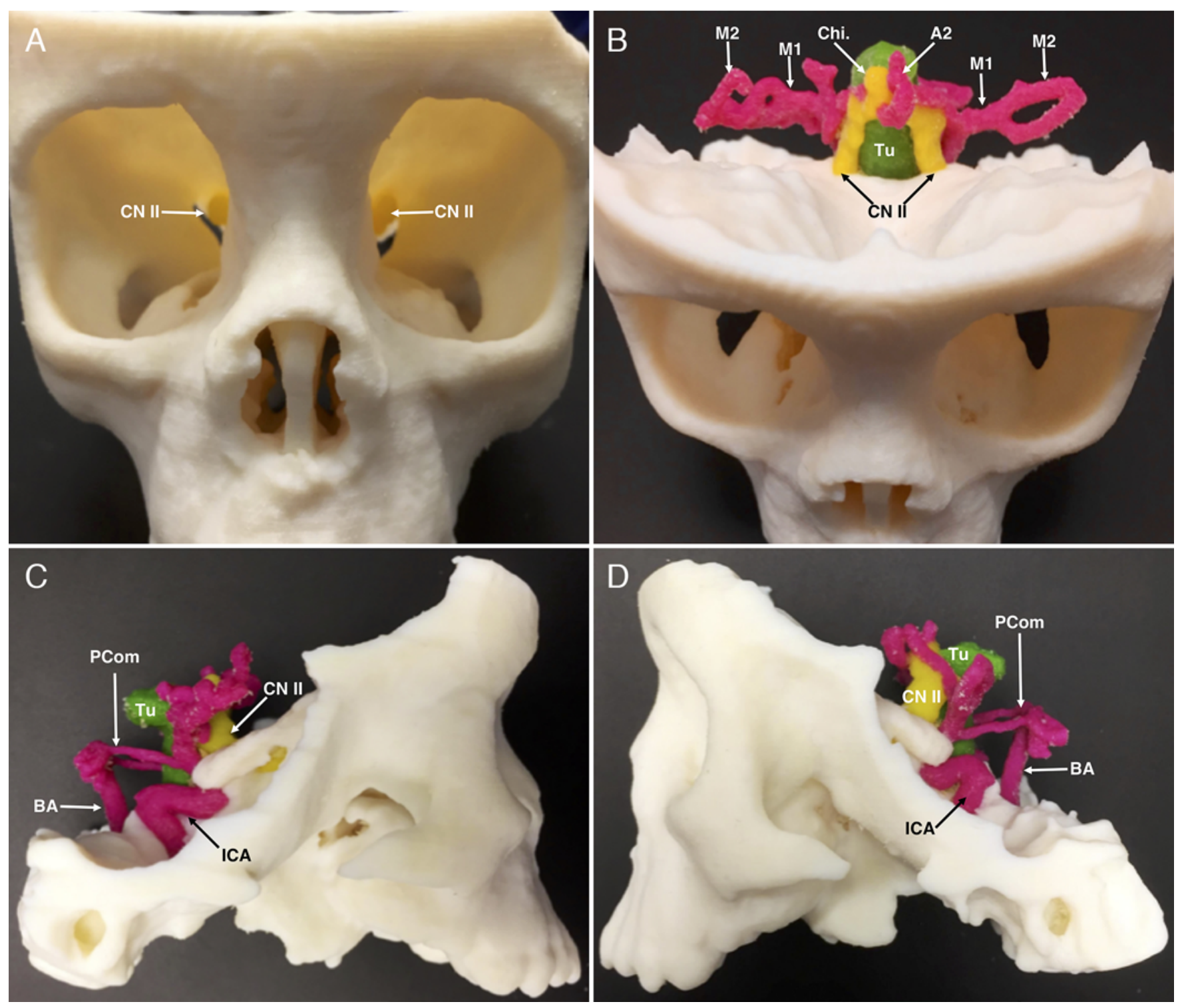

FIG. 8. Case 4. A: An anterior view of the 3D printed skull created for case 4. Notable from this view are the optic nerves (CN II; yellow) within the orbits. B: A superior-oblique view demonstrating the anterior and middle cranial fossae. The tumor (Tu; green) has vertically displaced both optic nerves (CN II) and the chiasm (Chi.), which sits on the anterosuperior aspect of the tumor. Also visible from this view are the vascular structures emanating from the circle of Willis, including the $A_{2}$ segment of the ACA and the bilateral $M_{1}$ and $M_{2}$ portions of the MCA. C: A right-sided view demonstrating middle and posterior fossae. Notable from this view are the vascular structures surrounding the tumor: the BA, which gives rise to the PCom. The terminal aspect of the ICA is also visualized well from this angle. D: A left-sided view demonstrating the middle and posterior cranial fossae. The same structures as visible in $\mathrm{C}$ are labeled in this view. Notable, however, is the extent of vertical displacement of CN II.

tient-specific, customized pedicle screw guidance devices to ensure the correct angle of screw placement during spine surgery ${ }^{5}$ and creation of models of parts, or the complete spine, together with pathology for surgical planning and patient education purposes. . $^{15,28}$

The relative advancement of VR and AR methods may also represent an alternative means of anatomical visualization for neurosurgeons. Like 3D printing, commercially available VR platforms are able to model anatomy and pathology utilizing conventional neuroimaging sequences like CT, MRI, etc., ${ }^{76,19}$ Due to the final "model" being a virtual 3D image, the potential of VR to model larger body areas is a definite advantage. VR is also not constrained by printing time, material cost, or the need to manually process the models following fabrication. Nevertheless, acquisition cost of VR hardware and software may be substantial and the virtual representation does not possess the tactile properties of 3D printed models. This may make VR models less beneficial for patient education and presurgical planning. At this stage, it is not known which technique is more advantageous for neurosurgeons, trainees, and patients. Further investigation and comparisons are warranted. 


\section{Conclusions}

3D printed models are beneficial in pre- and intraoperative planning to depict patient-specific anatomy and pathology relevant to skull base and neurovascular surgery. Due to their rapid fabrication framework, they are feasible for widespread use and may aid in the training of neurosurgeons and patient education.

\section{References}

1. Baskaran V, Štrkalj G, Štrkalj M, Di Ieva A: Current applications and future perspectives of the use of 3D printing in anatomical training and neurosurgery. Front Neuroanat 10:69, 2016

2. Evins AI, Dutton J, Imam SS, Dadi AO, Xu T, Cheng D, et al On-demand intraoperative 3-dimensional printing of custom cranioplastic prostheses. Oper Neurosurg (Hagerstown) 15:341-349, 2018

3. Galloway RL Jr, Maciunas RJ, Edwards CA II: Interactive image-guided neurosurgery. IEEE Trans Biomed Eng 39:1226-1231, 1992

4. Govsa F, Karakas AB, Ozer MA, Eraslan C: Development of life-size patient-specific 3D-printed dural venous models for preoperative planning. World Neurosurg 110:e141-e149, 2018

5. Guo F, Dai J, Zhang J, Ma Y, Zhu G, Shen J, et al: Individualized 3D printing navigation template for pedicle screw fixation in upper cervical spine. PLoS One 12:e0171509, 2017

6. Kikinis R, Gleason PL, Moriarty TM, Moore MR, Alexander E III, Stieg PE, et al: Computer-assisted interactive threedimensional planning for neurosurgical procedures. Neurosurgery 38:640-651, 1996

7. Kockro RA, Serra L: Patient-specific virtual reality simulation for minimally invasive neurosurgery, in Alaraj A (ed): Comprehensive Healthcare Simulation: Neurosurgery, Comprehensive Healthcare Simulation. Cham: Springer International Publishing, 2018, pp 159-184

8. Kono K, Shintani A, Okada H, Terada T: Preoperative simulations of endovascular treatment for a cerebral aneurysm using a patient-specific vascular silicone model. Neurol Med Chir (Tokyo) 53:347-351, 2013

9. Lumenta CB, Gumprecht H, Krammer MJ: Image-guided neurosurgery, in Lumenta CB, Di Rocco C, Haase J, et al (eds): Neurosurgery: European Manual of Medicine. Berlin: Springer, 2010, pp 579-590

10. Mashiko T, Otani K, Kawano R, Konno T, Kaneko N, Ito Y, et al: Development of three-dimensional hollow elastic model for cerebral aneurysm clipping simulation enabling rapid and low cost prototyping. World Neurosurg 83:351-361, 2015

11. Meola A, Cutolo F, Carbone M, Cagnazzo F, Ferrari M, Ferrari V: Augmented reality in neurosurgery: a systematic review. Neurosurg Rev 40:537-548, 2017

12. Michalski MH, Ross JS: The shape of things to come: 3D printing in medicine. JAMA 312:2213-2214, 2014

13. Namba K, Higaki A, Kaneko N, Mashiko T, Nemoto S, Watanabe E: Microcatheter shaping for intracranial aneurysm coiling using the 3-dimensional printing rapid prototyping technology: preliminary result in the first 10 consecutive cases. World Neurosurg 84:178-186, 2015

14. Panesar SS, Belo JTA, D'Souza RN: Feasibility of clinicianfacilitated three-dimensional printing of synthetic cranioplasty flaps. World Neurosurg 113:e628-e637, 2018

15. Park HJ, Wang C, Choi KH, Kim HN: Use of a life-size three-dimensional-printed spine model for pedicle screw instrumentation training. J Orthop Surg Res 13:86, 2018
16. Pelargos PE, Nagasawa DT, Lagman C, Tenn S, Demos JV, Lee SJ, et al: Utilizing virtual and augmented reality for educational and clinical enhancements in neurosurgery. J Clin Neurosci 35:1-4, 2017

17. Phan K, Sgro A, Maharaj MM, D’Urso P, Mobbs RJ: Application of a 3D custom printed patient specific spinal implant for C1/2 arthrodesis. J Spine Surg 2:314-318, 2016

18. Pucci JU, Christophe BR, Sisti JA, Connolly ES Jr: Threedimensional printing: technologies, applications, and limitations in neurosurgery. Biotechnol Adv 35:521-529, 2017

19. Qiu TM, Zhang Y, Wu JS, Tang WJ, Zhao Y, Pan ZG, et al: Virtual reality presurgical planning for cerebral gliomas adjacent to motor pathways in an integrated 3-D stereoscopic visualization of structural MRI and DTI tractography. Acta Neurochir (Wien) 152:1847-1857, 2010

20. Snyderman C, Kassam A, Carrau R, Mintz A, Gardner P, Prevedello DM: Acquisition of surgical skills for endonasal skull base surgery: a training program. Laryngoscope 117:699-705, 2007

21. Tai BL, Rooney D, Stephenson F, Liao PS, Sagher O, Shih AJ, et al: Development of a 3D-printed external ventricular drain placement simulator: technical note. J Neurosurg 123:1070-1076, 2015

22. Waran V, Narayanan V, Karrupiah R, Cham CY: 3D printing in neurosurgery, in Rybicki FJ, Grant GT (eds): 3D Printing in Medicine. Cham: Springer, 2017, pp 51-58

23. Waran V, Narayanan V, Karuppiah R, Owen SLF, Aziz T: Utility of multimaterial 3D printers in creating models with pathological entities to enhance the training experience of neurosurgeons. J Neurosurg 120:489-492, 2014

24. Waran V, Narayanan V, Karuppiah R, Pancharatnam D, Chandran H, Raman R, et al: Injecting realism in surgical training-initial simulation experience with custom 3D models. J Surg Educ 71:193-197, 2014

25. Weinstock P, Prabhu SP, Flynn K, Orbach DB, Smith E: Optimizing cerebrovascular surgical and endovascular procedures in children via personalized 3D printing. J Neurosurg Pediatr 16:584-589, 2015

26. Wilcox B, Mobbs RJ, Wu AM, Phan K: Systematic review of $3 \mathrm{D}$ printing in spinal surgery: the current state of play. $\mathbf{J}$ Spine Surg 3:433-443, 2017

27. Wurm G, Tomancok B, Pogady P, Holl K, Trenkler J: Cerebrovascular stereolithographic biomodeling for aneurysm surgery. Technical note. J Neurosurg 100:139-145, 2004

28. Xu N, Wei F, Liu X, Jiang L, Cai H, Li Z, et al: Reconstruction of the upper cervical spine using a personalized 3Dprinted vertebral body in an adolescent with Ewing sarcoma. Spine (Phila Pa 1976) 41:E50-E54, 2016

\section{Disclosures}

The authors report no conflict of interest concerning the materials or methods used in this study or the findings specified in this paper.

\section{Author Contributions}

Conception and design: Panesar. Analysis and interpretation of data: Panesar. Drafting the article: Panesar, Mukherjee, Abhinav. Critically revising the article: all authors. Reviewed submitted version of manuscript: all authors.

\section{Correspondence}

Juan C. Fernandez-Miranda: Stanford University, Palo Alto, CA. drjfm@stanford.edu. 